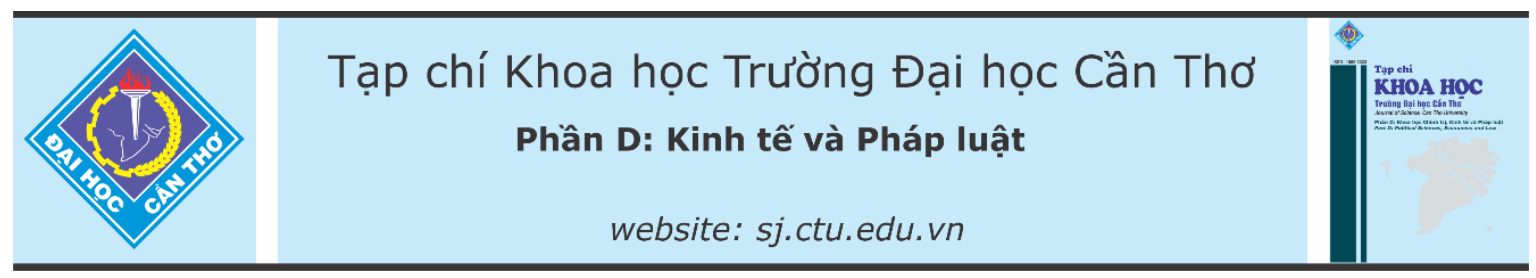

DOI:10.22144/ctu.jvn.2021.132

\title{
NGHIÊN CÚU SỬ HÀI LÒNG CỦA NGƯờI HỌC ĐỐI VỚI HÌNH THỨC HỌC TẬP TRỰC TUYẾN (E-LEARNING): TRƯỜNG HỢP SINH VIÊN NGÀNH KINH TẾ TẠI THÀNH PHỐ HỒ CHÍ MINH
}

\author{
Lê Nam Hải* và Trần Yến Nhi \\ Khoa Quản trị kinh doanh, Truờng Đại học Công nghiệp Thành phố Hồ Chi Minh \\ *Người chịu trách nhiệm về bài viết: Lê Nam Hải (email: lenamhai@iuh.edu.vn)
}

\section{Thông tin chung:}

Ngày nhận bài: 10/03/2021

Ngày nhận bài sưa: 06/05/2021

Ngày duyệt đăng: 20/08/2021

\section{Title:}

Students' satisfaction on elearning platform: The case of economic student in $\mathrm{Ho} \mathrm{Chi}$ Minh city

\section{Tù khóa:}

Học tập trục tuyến, mô hình hệ thống thông tin thành công, mô hình chấp nhận công nghệ, sự hài lòng

\section{Keywords:}

E-learning services, satisfaction, successful information system, technology adoption model

\begin{abstract}
The objective of this study is to analyze the factors affecting the students' satisfaction with e-learning services. Technology adoption model and successful information system theory are applied to propose model and test hypotheses. The data were collected from 267 students who studied via e-learning platform at some universities in Ho Chi Minh City. The results show that perceived ease of use, perceived usefulness, information quality, system quality, instructor, support services, subjective norm, and perceived behavior control positively affects learners' satisfaction. In addition, implications were proposed for educational institutions to enhance students' satisfaction through enhancing the quality of $e$ learning services.
\end{abstract}

\section{TÓM TẮT}

Mục tiêu của nghiên cưu này là phân tích nhũng nhân tố ảnh hưởng đến sư hài lòng của người học đối với dịch vu họ tập trục tuyến (e-learning). Nghiên cưu vận dụng mô hình chấp nhận công nghệ và lý thuyết hệ thông thông tin thành công làm cơ sở đề xuất và kiểm định giả thuyết. Dũ liệu phân tích được thu thập tì 267 sinh viên hiện đang theo học tại một số truoòng đại hoc ở thành phố Hồ Chí Minh và đã tùng tham gia dịch vụ học tập trực tuyến. Kết quả cho thấy nhận thức dễ sử dụng, nhận thức sụ hữu ich, chất luợng thông tin, chất luợng hệ thống, giảng viên huớng dẫn, dịch vu hỗ trợ, chuấn chủ quan và nhận thức kiểm soát hành vi có ảnh hương tích cực đến sự hài lòng của người học đối với hình thức hoc tập trực tuyến. Ngoài ra, một số hàm ý quản trị được thảo luận và đề xuất nhằm giúp cho các tổ chức giáo dục gia tăng sự hài lòng của người học thông qua việc nâng cao chất luợng dịch vu học tập trực tuyến.

\section{GIỚI THIỆ}

Sự phát triển mạnh mẽ của công nghệ thông tin cùng với sự bùng nổ của internet đã mang lại nhiều thay đổi đáng kể trong hoạt động và quản lý ở nhiều lĩnh vực khác nhau như: y tế, giáo dục - đào tạo, quốc phòng - an ninh, công nghiệp, thương mại, dịch vụ. Điển hình trong lĩnh vực giáo dục - đào tạo, bên cạnh hình thức học tập truyền thống, hình thức học tập trực tuyến ra đời đã mang lại nhiều lợi ích cho cả người dạy và người học. Học trực tuyến (elearning) là hình thức học tập thông qua một máy vi tính, điện thoại thông minh có kết nối mạng internet. Từ đó, người học có thể tiết kiệm được thời gian chi phí vì hình thức học này giúp giải quyết rào cản về 
khoảng cách thời gian và địa lý. Việc giao tiếp giữa người học và người hướng dẫn được thực hiện thông qua các chức năng đa phương tiện khác nhau. Hình thức học tập này ra đời cho phép giảng viên có thể cập nhật các nội dung đào tạo thường xuyên hơn, có thể kiểm soát được lượng kiến thức mà người học nhận được qua các buổi học bằng hệ thống tự đánh giá, tài liệu học tập được gửi ở các dạng tập tin khác nhau so với hình thức giảng dạy truyền thống. Hình thức này đang được áp dụng khá phổ biến ở nhiều nước phát triển và đang phát triển trên thế giới như Mỹ, Anh, Hàn Quốc, Trung Quốc, Nhật Bản, Ân Độ (Kentnor, 2015; Harasim, 2006; Innovation Center Denmark Seoul, 2014). Đặc biệt trong bối cảnh phòng chống dịch bệnh viêm đường hô hấp cấp SARS-CoV-2 (COVID-19), các nước trên thế giới nói chung và Việt Nam nói riêng đang thực hiện các biện pháp thắt chặt như hạn chế tiếp xúc trực tiếp gần giữa người với người, tránh tụ tập đông người thậm chí là thực hiện giãn cách, tạm ngừng một số hoạt động hoặc tạm thời đóng cửa các dịch vụ không thiết yếu,...nhằm ngăn chặn sự lây lan của dịch bệnh thì hình thức hoc tập trực tuyến được xem là phương thức hữu hiệu và cần thiết để người học có thể cập nhật kiến thức mà không cần phải học tập tập trung tại trường, tránh châm hoặc trễ chương trình đào tạo (Bộ Giáo dục và Đào tạo, 2020).

Tại Việt Nam, hình thức học trực tuyến cũng đã có sự phát triển khá mạnh mẽ, nhiều đơn vị đã tạo ra các trang web học trực tuyến như Violet.vn, Hocmai.vn, Topica thu hút được sự quan tâm của nhiều người dùng. Tính đến năm 2015 tại Việt Nam có hơn 100 đơn vị đã áp dụng và dạy học theo phương thức giáo dục trực tuyến này. Đồng thời, mô hình giáo dục này hiện nay được tập trung nhiều vào giáo dục đại học thông qua hệ thống website của nhà trường. Theo Vũ Hữu Đức (2020), trong đào tạo từ xa học trực tuyến phát triển với hình thức là chương trình đào tạo cấp bằng đại học, còn trong đào tạo chính quy học trực tuyến được sử dụng là một hình thức hỗ trợ hoặc kết hợp song song với hình thức học truyền thống. Với khoảng 460.000 sinh viên đang theo học tại các trường với các hình đào tạo khác nhau, Thành phố Hồ Chí Minh cũng là một trong những trung tâm giáo dục lớn, trong đó số lượng trường học đào tạo chính quy đại học là 60 trường, với 46 trường công lập và 14 trường ngoài công lập (Tổng cục Thống kê, 2018). Vì vậy, việc quan tâm và áp dụng hình thức học trực tuyến song song với phương thức học truyền thống tại các cơ sở giáo dục đại học tại thành phố này là cần thiết không chỉ thể hiện sự đổi mới trong đào tạo và còn có ý nghĩa thực tiễn trong bối cảnh phòng, chống dịch bệnh.

Vấn đề đặt ra là, bên cạnh việc triển khai áp dụng hình thức học tập trực tuyến thì cần có sự quan tâm đến phản hồi, cảm nhận đánh giá và sự hài lòng của người học đối với hình thức này với mục tiêu nâng cao hơn nữa chất lượng dịch vụ đào tạo và khắc phục những hạn chế còn tồn tại nếu có. Ở khía cạnh này, một số học giả cũng đã tiến hành nghiên cứu các nhân tố nào sẽ ảnh hưởng đến sự hài lòng của người học đối với hình thức học trực tuyến. Điển hình như nghiên cứu của Chiu et al. (2005), Roca et al. (2006), Sun et al. (2008), Wu et al. (2008), Tarhini et al. (2013), Vũ Thúy Hằng và Nguyễn Mạnh Tuân (2013), Mohammadi (2015), Pham et al. (2019). Các nghiên cứu này được thực hiện hầu hết là ở các nước phát triển như Đài Loan, Hoa Kỳ, Hàn Quốc, Trung Quốc, Tây Ban Nha,.... Các lý thuyết được các tác giả vận dụng để giải thích trong một số nghiên cứu đi trước bao gồm: lý thuyết về phân biệt kỳ vọng EDT (expectancy disconfirmation theory), mô hình chấp nhận công nghệ TAM (technology acceptance model) và hệ thống thông tin thành công ISS (Information systems success). Trong khi đó, tại Việt Nam vẫn còn tương đối ít nhà nghiên cứu tập trung vào việc áp dụng kết hợp mô hình hệ thống thông tin thành công ISS và mô hình chấp nhận công nghệ TAM để xem xét sự hài lòng của người học dựa trên cảm nhận của họ từ việc trãi nghiệm hình thức học tập trực tuyến. Vì vậy, bài nghiên cứu này sẽ xem xét các nhân tố nào có ảnh hưởng đến sự hài lòng, mức độ hài lòng của sinh viên khi sử dụng hình thức học tập trực tuyến dựa trên nền tảng của hai mô hình đã đề cập. Ngoài ra, một số đề xuất cũng được đưa ra nhằm giúp các tổ chức giáo dục tại Việt Nam nói chung và Thành phố Hồ Chí Minh nói riêng có thể cải thiện và nâng cao chất lượng dịch vụ đào tạo của loại hình này.

\section{PHƯONG PHÁP NGHIÊN CÚU}

\subsection{Tổng quan tài liệu và mô hình nghiên cứu}

Mô hình chấp nhận công nghệ TAM của Davis (1989) và mô hình hệ thống thông tin thành công IS của DeLone and McLean (1992) được vận dụng làm nền tảng để giải thích và biện luận mô hình nghiên cứu. Theo đó, nhận thức dễ sử dụng và nhận thức sự hữu ích trong mô hình TAM là hai nhân tố quan trọng ảnh hưởng đến việc chấp nhận sử dụng hệ thống thông tin điện tử. Hai yếu tố này cũng được nhiều học giả sử dụng để đo lường sự hài lòng của người học đối với hình thức học trực tuyến như Chiu et al. (2005), Roca et al. (2006), Sun et al. (2008), 
Wu et al. (2008), Tarhini et al. (2013), Mohammadi (2015). Cùng với đó, mô hình hệ thống thông tin thành công IS được DeLone and McLean phát triển vào năm 1992 và mở rộng hiệu chỉnh vào năm 2003 đã thể hiện quan điểm khác nhau về đánh giá hệ thống thông tin. Cụ thể, sáu yếu tố trong mô hình của DeLone and McLean (2003) là: chất lượng hệ thống, chất lượng thông tin, chất lượng dịch vụ, ý định sử dụng và sử dụng hệ thống, sự hài lòng của người sử dụng, tác động thuần. Vì vậy, khi xem xét trong bối cảnh triển khai hình thức học tập trực tuyến tại Việt Nam cùng với việc kế thừa một số nghiên cứu đi trước của Roca et al. (2006), Sun et al. (2008), Wu et al. (2008), Tarhini et al. (2013), Pham et al. (2019), nghiên cứu này đề xuất các giả định cơ bản rằng sự hài lòng của người học sẽ bị tác động bởi các nhân tố là nhận thức dễ sử dụng, nhận thức sự hữu ích, chất lượng thông tin, chất lượng hệ thống; giảng viên hướng dẫn; dịch vụ hỗ trợ; chuẩn chủ quan và nhận thức kiểm soát hành vi (Hình 1).

Nhận thức dễ sử dụng: là "mức độ mà một cá nhân đó tin rằng việc sử dụng một hệ thống cụ thể nào đó sẽ không tốn quá nhiều công sức của họ" (Davis,1989). Chiu et al. (2005), Roca et al. (2006), Sun et al. (2008), Wu et al. (2008), Tarhini et al. (2013) đã nghiên cứu và đề cập đến khả năng dễ sử dụng của một hệ thống học tập trực tuyến đối với sinh viên thông qua cách sử dụng hệ thống, cách điều khiển các chức năng hay các thao tác trên hệ thống có sự tác động tích cực đến sự hài lòng của sinh viên khi sử dụng hình thức học trực tuyến. Theo Sun et al. (2008), "nhận thức dễ sử dụng trong một hệ thống dịch vụ học trực tuyến (e-learning) là nhận thức của người học về sự dễ dàng cho việc sử dụng nó". Vì vậy, nhận thức dễ sử dụng được xem là có ảnh hưởng đến sự hài lòng của sinh viên:

H1: Nhận thức dễ sư dụng có ảnh hưởng tích cực đến sụ hài lòng của sinh viên khi sử dụng hình thức hoc trục tuyến.

Nhận thức sự hữu ích: là việc mà một cá nhân tin rằng sử dụng một hệ thống đặc thù sẽ nâng cao sự hiệu quả của họ (Davis, 1989). Trong một nghiên cứu của Roca et al. (2006) đã có đề cập đến nhân tố nhận thức sử hữu ích và kết quả đạt được qua nghiên cứu là tính hữu dụng có tác động lớn nhất đến sự hài lòng. Có sự giả định rằng tính hữu ích của một hệ thống càng cao về phương tiện, trang web, chức năng thì thái độ của người sử dụng đối với dịch vụ càng tích cực hơn (Sun et al., 2008). Tarhini et al. (2013) và Mohammadi (2015) cũng đã đề cập đến tính hữu ích có sự ảnh hưởng tích cực đến ý định hành vi sử dụng hệ thống học tập dựa trên web. Khi người dùng nhận thấy học tập trực tuyến sẽ hữu ích trong việc đạt được kiến thức và các kỹ năng mong muốn, họ có nhiều khả năng sẽ sử dụng hệ thống (Cheok \& Wong, 2015). Do đó, giả thuyêt được đưa ra rằng:

H2: Nhận thức sụ hũu ích có ảnh hưởng tích cực đến sụ hài lòng của sinh viên khi sử dụng hình thức học trục tuyến.

Chất lượng thông tin: là sự liên quan đến chất lượng đầu ra của một thực thể, chất lượng thông tin thường mang tích kịp thời, phạm vi, mức độ liên quan và mức độ chính xác của thông tin được tạo bởi một hệ thống thông tin (Delone \& McLean, 1992). Roca et al. (2006) và Mohammadi (2015) cho thấy chất lượng thông tin có sự tác động tích cực đến sự hài lòng của sinh viên đối với hình thức học trực tuyến. Thêm vào đó, Pham et al. (2019) cũng đã từng đề cập đến chất lượng thông tin là yếu tố để đánh giá chất lượng dịch vụ của một hệ thống có ảnh hưởng đến sự hài lòng của người dùng thông qua chất lượng tài liệu của khóa học, tài liệu học tập phải có lý thuyết và thực tế, đảm bảo tính cập nhật liên tục và liên quan đến chương trình đào tạo. Riêng trong nghiên cứu của Vũ Thúy Hằng và Nguyễn Mạnh Tuân (2013) thì yếu tố về thiết kế nội dung cần phải rõ ràng, thông tin dễ lấy và dễ tiếp cận, các hình thức hoàn thành những bài tập được giao có sự đa dạng thể loại từ đó tạo cho sinh viên sự thích thú khi học và cảm giác hài lòng. Chất lượng thông tin sẽ đánh giá được lượng kiến thức mà sinh viên có được sau mỗi khóa học có phù hợp, kịp thời, đáp ứng đúng với mục đích của sinh viên không, do đó có sự liên quan mật thiết và có ý nghĩa giữa chất lượng thông tin với sự hài lòng của sinh viên (Chiu et al., 2005; Sun et al., 2008).

H3: Chất lương thông tin có ảnh hưởng tích cưc đến sự hài lòng của sinh viên khi sủ dụng hình thức học trục tuyến.

Chất lượng hệ thống: có sự liên quan đến việc có "lỗi" trong hệ thống hay không, tính nhất quán của giao diện người dùng, dễ sử dụng, tỷ lệ phản hồi trong các hệ thống tương tác, chất lượng tài liệu, chất lượng và khả năng duy trì (Seddon, 1997). Roca et al. (2006) và Mohammadi (2015) chỉ ra rằng chất lượng hệ thống có ảnh hưởng tích cực đến sự hài lòng của người sử dụng. Pham et al. (2019) cho rằng chất lượng hệ thống học tập trực tuyến được thể hiện thông qua trang web học trực tuyến của các trường đại học. Wu el al. (2008) cũng cho rằng chức năng hệ thống là khả năng nhận thức của sinh viên về hệ thống như việc cung cấp quyền truy cập linh hoạt vào các chương trình đào tạo và cho phép sinh viên 
truy cập tài liệu, nội dung khóa học, bài tập về nhà, hoàn thành bài kiểm tra một cách dễ dàng có ảnh hưởng tích cực đến sự hài lòng của sinh viên thông qua giá trị cảm nhận khi sử dụng dịch vụ. Theo Vũ Thúy Hằng và Nguyễn Mạnh Tuân (2013), chất lượng hệ thống là việc người học dễ dàng truy cập vào từng chức năng nội dung bài giảng tại bất kì thời điểm và địa điểm trong quá trình học tập. Ngoài ra, sinh viên cũng có sự nhạy cảm với các công cụ công nghệ được sử dụng ở hệ thống trong quá trình học, sinh viên đưa ra cảm nhận về sự phù hợp của nền tảng và thiết kế của nó, bao gồm các tính năng như truy cập vào nội dung, sự rõ ràng trong các bản trình bày, tốc độ tải xuống của văn bản hoặc hình ảnh đồ họa, video (Martín-Rodríguez et al., 2015).

H4: Chất lương hệ thống có sụ ảnh hưởng tích cực đến sụ hài lòng của sinh viên khi sủ dụng hình thức hoc trực tuyến.

Giảng viên hướng dẫn: giảng viên là người giảng dạy, giao tiếp với các học viên trong lớp học, quản lý khóa học và học viên trong lớp của mình; biên soạn bài giảng, bài tập (Huỳnh Đệ Thủ, 2019). Ngoài ra, họ là người định hướng, người hỗ trợ sinh viên trong quá trình học tập qua dịch vụ (Bùi Kiên Trung, 2016). Vì vậy, giảng viên hướng dẫn phải có cả kiến thức lý thuyết và thực tiễn, luôn quan tâm đến sở thích của sinh viên, và thúc đẩy sự tương tác liên tục của sinh viên (Pham et al., 2019). Đồng thời, giảng viên được xem là nguồn nhân lực quan trọng nhất trong việc để cung cấp cho sinh viên một chất lượng giáo dục hoàn toàn mới (Cheok \& Wong, 2015). Nghiên cứu của Roca et al. (2006) cũng đã có đề cập đến giảng viên hướng dẫn có sự tác động đến sự hài lòng. Theo đó, có 3 khía cạnh để đo lường chất lượng đội ngũ giảng viên bao gồm: chuyên môn của giảng viên, sự tương tác của giảng viên đối với sinh viên và sự hỗ trợ kịp thời của giảng viên có tác động tích cực đến sự hài lòng của sinh viên.

H5: Giảng viên hướng dẫn có sự ảnh huơong tích cực đến sụ hài lòng của sinh viên khi sử dụng hình thức hoc trục tuyến.

Dịch vụ hỗ trợ: là một phần trong chất lượng dịch vụ, chất lượng dịch vụ không tập trung hoàn toàn vào đánh giá quá trình dạy và học mà còn có sự đánh giá về các dịch vụ hành chính và các dịch vụ bổ sung khác (Martínez-Argüelles \& BatallaBusquets, 2016 ). Theo nghiên cứu của Pham et al. (2019), chất lượng dịch vụ học tập trực tuyến được đánh giá qua khía cạnh về dịch vụ hỗ trợ hoặc dịch vụ hành chính và xem xét nó như một nhân tố có ảnh hưởng tích cực đến sự hài lòng của sinh viên khi sử dụng dịch vụ. Việc quản lý các khía cạnh của các dịch vụ hỗ trợ này là không thể thiếu để đảm bảo sự trung thành của sinh viên (Martínez-Argüelles \& Batalla-Busquets, 2016).

H6: Dịch vu hỗ trợ có sự ảnh hưởng tích cực đến sư hài lòng của sinh viên khi sư dụng hình thức học trực tuyến.

Chuẩn chủ quan: là nhận thức của một người nào đó cho rằng hầu hết những người quan trọng đối với họ cho rằng họ nên hay không nên thực hiện một điều gì đó (Ajzen, 1991). Roca et al. (2006) đã đưa nhân tố chuẩn chủ quan này vào mô hình để giải thích cho sự ảnh hưởng từ các tác nhân bên ngoài từ người khác khi sử dụng dịch vụ học tập trực tuyến. Thêm vào đó, Tarhini et al. (2013) cũng có đề cập đến sự ảnh hưởng tích cực của chuẩn chủ quan đến sự hài lòng.

H7: Chuẩn chủ quan có sự ảnh hương tích cực đến sụ hài lòng của sinh viên khi sủ dụng hình thức hoc trực tuyến.

Nhận thức kiểm soát hành vi: yếu tố này được xem xét ở hai khía cạnh là sự hiệu quả của máy tính và sự hiệu quả của internet. Đó là việc mà một cá nhân tin rằng mình có khả năng tự sử dụng và nhận thấy sự dễ sử dụng của máy tính và internet sẽ có tác động tích cực đến sự hài lòng khi sử dụng hình thức học trực tuyến (Roca et al., 2006). Sự hiệu quả của máy tính được xác nhận là yếu tố quyết định sự chấp nhận và sử dụng đối với hệ thống (Wu et al., 2010). Roca et al. (2006) đã đề cập trong nghiên cứu rằng sự hiệu quả là niềm tin của một cá nhân nghĩ rằng bản thân có thể thực hiện một việc làm gì đó hay một hành vi cụ thể. Nói cách khác, đó là năng lực của bản thân là sự đánh giá bản thân về khả năng của mình đối với một khóa học cụ thể (Isik, 2008). Wu et al. (2010) xác định tính hiệu quả của máy tính là sự tự tin về khả năng hoàn thành các nhiệm vụ học tập hoặc các bài tập bằng hệ thống dịch vụ học trực tuyến của một người.

H8: Nhận thức kiểm soát hành vi có sư ảnh hưởng tích cưc đến sụ hài lòng của sinh viên khi sủ dụng hình thức học trục tuyến. 


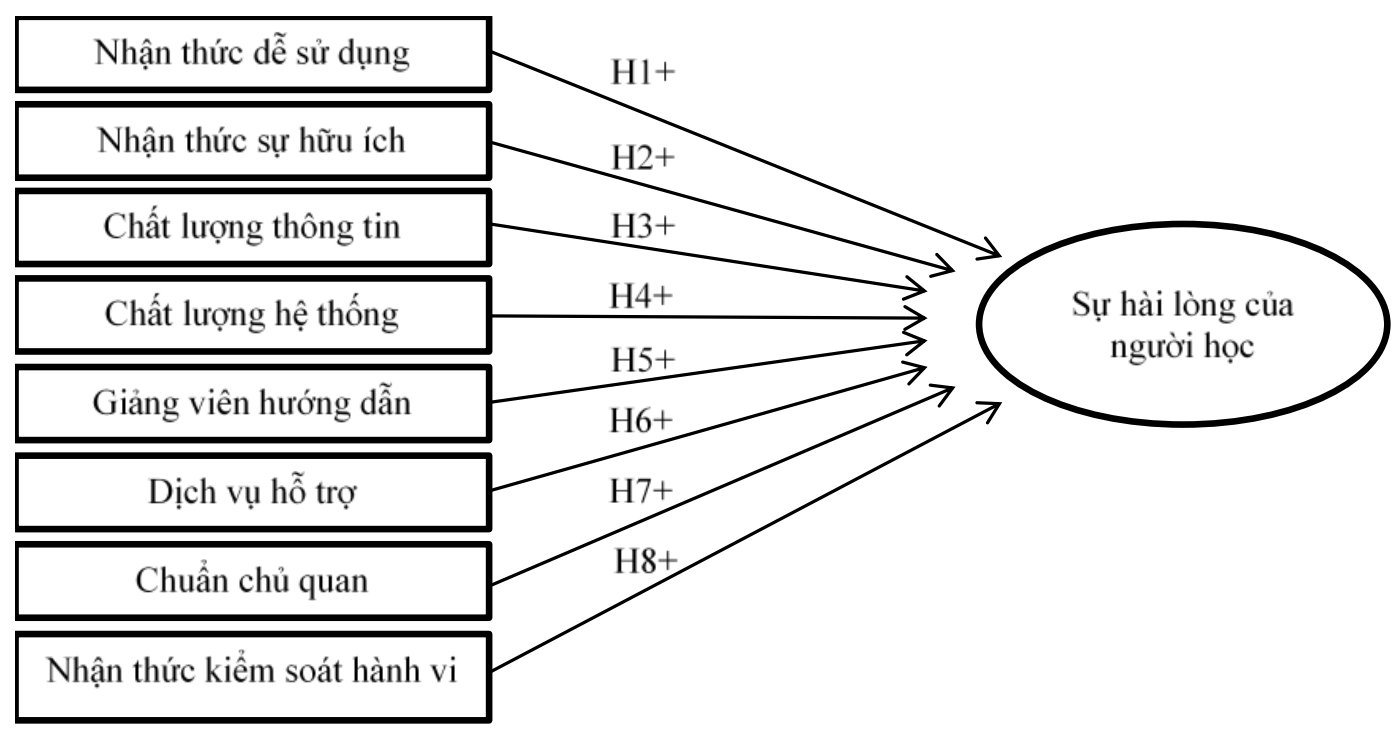

Hình 1. Mô hình nghiên cứu đề xuất

\subsection{Thang do}

Các giả thuyết nghiên cứu được kiểm định thông qua bộ dữ liệu sơ cấp với 267 quan sát hợp lệ được thu thập bằng phương pháp lấy mẫu phi xác suất theo kiểu thuận tiện. Các đối tượng được khảo sát là sinh viên khối ngành kinh tế tại một số trường đại học ở thành phố Hồ Chí Minh (TP.HCM) đã và đang sử dụng hình thức học trực tuyến cho quá trình học tập và nghiên cứu của mình. Bộ thang đo chính thức gồm 47 mục hỏi được kế thừa và hiệu chỉnh từ các nghiên cứu đi trước có liên quan thông qua phỏng vấn sâu và lấy ý kiến chuyên gia (Bảng 1).

Bảng 1. Xây dựng thang đo cho các nhân tố nghiên cứu trong mô hình

\begin{tabular}{|c|c|c|}
\hline Mã hóa & $\begin{array}{l}\text { Biến quan sát của các nhân tố } \\
\text { Nhận thức dễ sử dụng (Perceived Ease of Use) - PE }\end{array}$ & -Nguồn \\
\hline PE1 & Học cách sử dụng dịch vụ học trực tuyến đối với tôi thật dễ dàng & $\begin{array}{l}\text { Roca et al. (2006), } \\
\text { Wu et al. (2008) }\end{array}$ \\
\hline PE2 & $\begin{array}{l}\text { Tôi dễ dàng thực hiện những gì tôi muốn làm trên hệ thống dịch vụ } \\
\text { học tập trực tuyến }\end{array}$ & $\begin{array}{l}\text { Wu et al. (2008), } \\
\text { Sun et al. (2008) }\end{array}$ \\
\hline PE3 & $\begin{array}{l}\text { Dịch vụ học trực tuyến giúp tôi dễ dàng trở nên thành thạo hơn khi sử } \\
\text { dụng }\end{array}$ & Roca et al. (2006) \\
\hline PE4 & $\begin{array}{l}\text { Sự tương tác của tôi đối với dịch vụ học trực tuyến thì rõ ràng và dễ } \\
\text { hiểu }\end{array}$ & $\begin{array}{l}\text { Tarhini et al. (2013), } \\
\text { Roca et al. (2006) }\end{array}$ \\
\hline PE5 & Tôi thấy hệ thống học trực tuyến dễ sử dụng & $\begin{array}{l}\text { Tarhini et al. (2013), } \\
\text { Sun et al. (2008) }\end{array}$ \\
\hline \multirow[t]{2}{*}{ PE6 } & $\begin{array}{l}\text { Trang web của khóa học trực tuyến cho phép tôi tìm kiếm thông tin dễ } \\
\text { dàng }\end{array}$ & \multirow[t]{2}{*}{$\tilde{\mathrm{e}}_{\text {Pham et al. (2019) }}$} \\
\hline & Nhận thức sự hữu ích (Perceived Usefulness) - PU & \\
\hline PU1 & $\begin{array}{l}\text { Sư dụng dịch vụ học trực tuyen co the cal thıẹn dược hıẹu suat học tạp } \\
\text { của tôi }\end{array}$ & $\begin{array}{l}\text { Roca et al. (2006), } \\
\text { Tarhini et al. (2013) }\end{array}$ \\
\hline PU2 & Sử dụng dịch vụ học trực tuyến sẽ nâng cao hiệu quả học tập của tôi & Roca et al. (2006) \\
\hline PU3 & Tôi thây dịch vụ học trực tuyến hữu ích cho việc học của tôi & Roca et al. (2006) \\
\hline PU4 & $\begin{array}{l}\text { Sử dụng dịch vụ học trực tuyến sẽ cho phép tôi hoàn thành việc học } \\
\text { tập nhanh hơn }\end{array}$ & Tarhini et al. (2013) \\
\hline PU5 & Sử dụng dịch vụ học trực tuyến giúp tôi tiết kiệm thời gian & Mohammadi (2015) \\
\hline PU6 & Sử dụng dịch vụ học trực tuyến giúp tôi tiết kiệm chi phí & Mohammadi (2015) \\
\hline & Chất lượng thông tin (Information Quality) - IN & \\
\hline
\end{tabular}




\begin{tabular}{|c|c|c|}
\hline Mã hóa & $\begin{array}{l}\text { Biến quan sát của các nhân tố } \\
\text { Nhận thức dễ sử dụng (Perceived Ease of Use) - PE }\end{array}$ & -Nguồn \\
\hline IN1 & Dịch vụ học trực tuyến cung cấp thông tin gây khó hiểu cho tôi & Roca et al. (2006) \\
\hline IN2 & $\begin{array}{l}\text { Thông tin từ dịch vụ học trực tuyến luôn được cập nhật cho mục đích } \\
\text { của tôi }\end{array}$ & Roca et al. (2006) \\
\hline IN3 & Độ tin cậy của thông tin đầu ra từ dịch vụ học trực tuyến cao & Roca et al. (2006) \\
\hline IN4 & Dịch vụ học trực tuyến cung cấp thông tin tôi cần kịp thời & Roca et al. (2006) \\
\hline IN5 & $\begin{array}{l}\text { Dịch vụ học trực tuyến không cung cấp đầy đủ thông tin cho mục đích } \\
\text { của tôi }\end{array}$ & Roca et al. (2006) \\
\hline \multirow[t]{2}{*}{ IN6 } & $\begin{array}{l}\text { Trang web của khóa học trực tuyến cung cấp cho tôi các thông tin có } \\
\text { giá trị }\end{array}$ & Pham et al. (2019) \\
\hline & Chất lượng hệ thống (System Quality) - SY & \\
\hline SY1 & $\begin{array}{l}\text { Dịch vụ học trực tuyến cho phép tôi kiểm soát các hoạt động học tập } \\
\text { của tôi }\end{array}$ & Wu et al. (2008) \\
\hline SY2 & $\begin{array}{l}\text { Hệ thống dịch vụ học trực tuyến có sự phản hồi nhanh chóng trong } \\
\text { thời gian cao điểm }\end{array}$ & Roca (2006) \\
\hline SY3 & $\begin{array}{l}\text { Việc sắp xếp thông tin hiển thị trên giao diện của hệ thống học trực } \\
\text { tuyến rât rõ ràng }\end{array}$ & Roca (2006) \\
\hline SY4 & $\begin{array}{l}\text { Các bước để hoàn thành việc học tập của tôi trên hệ thống học trực } \\
\text { tuyến là không đơn giản }\end{array}$ & Roca et al. (2006) \\
\hline \multirow[t]{2}{*}{ SY5 } & $\begin{array}{l}\text { Tôi cảm thấy an toàn khi cung cấp các thông tin bảo mật cho trang } \\
\text { web học trực tuyến tại trường đại học của tôi }\end{array}$ & Pham et al. (2019) \\
\hline & Giảng viên hướng dần (Instructors dimension) - ID & \\
\hline ID1 & $\begin{array}{l}\text { Giảng viên hướng dẫn học trực tuyến của tôi có kiến thức chuyên } \\
\text { môn }\end{array}$ & Pham et al. (2019) \\
\hline ID2 & $\begin{array}{l}\text { Giảng viên hướng dẫn học trực tuyến của tôi cung cấp các bài giảng } \\
\text { hay }\end{array}$ & Pham et al. (2019) \\
\hline ID3 & $\begin{array}{l}\text { Giảng viên hướng dẫn học trực tuyến của tôi cung cấp một môi } \\
\text { trường học tập khuyến khích sự tham gia tương tác }\end{array}$ & Pham et al. (2019) \\
\hline ID4 & $\begin{array}{l}\text { Tôi nhận được các đánh giá về bài tấp hoặc bài kiểm tra cho khóa học } \\
\text { một câch kị̂p thời từ giảng viên hướng dân học trực tuyến của tôi }\end{array}$ & Sun et al. (2008) \\
\hline \multirow[t]{2}{*}{ ID5 } & $\begin{array}{l}\text { Giảng viên hướng dẫn học trực tuyến của tôi có sự chuẩn bị tốt và có } \\
\text { tổ chức phù hợp đối với các khóa học }\end{array}$ & Roca et al. (2006) \\
\hline & Dịch vụ hỗ trợ (Support Service) - SS & \\
\hline SS1 & $\begin{array}{l}\text { Đối với việc học trực tuyến của tôi, trường đại học của tôi có dịch vụ } \\
\text { hồ trợ học trực tuyến ngay lần đầu sử dụng }\end{array}$ & Pham et al. (2019) \\
\hline SS2 & $\begin{array}{l}\text { Đối với việc học trực tuyến của tôi, nhân viên tại trường đại học sẽ } \\
\text { cho tôi biết chính xác các yêu cầu của tôi sẽ được thực hiện khi nào }\end{array}$ & Pham et al. (2019) \\
\hline SS3 & $\begin{array}{l}\text { Đối với việc học trực tuyến của tôi, nhân viên tại trường đại học của } \\
\text { tôi cung cấp cho tôi dịch vụ hố trợ nhanh chóng }\end{array}$ & Pham et al. (2019) \\
\hline SS4 & $\begin{array}{l}\text { Đối với việc học trực tuyến của tôi, nhân viên tại trường đại học của } \\
\text { tôi luôn hiểu được các nhu cầu cụ thể của tôi }\end{array}$ & Pham et al. (2019) \\
\hline \multirow[t]{2}{*}{ SS5 } & $\begin{array}{l}\text { Đối với việc học trực tuyến của tôi, dịch vụ hỗ trợ tại trường đại học } \\
\text { của tôi có giờ hoạt động thuận tiện }\end{array}$ & Pham et al. (2019) \\
\hline & Chuẩn chủ quan (Subjective Norm) - SN & \\
\hline SN1 & Bạn bè tôi nghĩ tôi nên sử dụng dịch vụ học trực tuyến & $\begin{array}{l}\text { Roca et al. (2006), } \\
\text { Tarhini et al. (2013) }\end{array}$ \\
\hline SN2 & $\begin{array}{l}\text { Tôi đã đọc các báo cáo tin tức rằng việc sử dụng dịch vụ học trực } \\
\text { tuyến là một phương pháp học tốt }\end{array}$ & Roca et al. (2006) \\
\hline SN3 & $\begin{array}{l}\text { Giáo viên hướng dấn của tôi nghĩ rằng tôi nên sử dụng dịch vụ học } \\
\text { trực tuyến vào quá trình học của mình }\end{array}$ & $\begin{array}{l}\text { Roca et al. (2006), } \\
\text { Tarhini et al. (2013) }\end{array}$ \\
\hline SN4 & Gia đình tôi nghĩ rằng tôi nên sử dụng dịch vụ học trực tuyến & Roca et al. (2006) \\
\hline & Nhận thức kiểm soát hành vi (Perceived Behavior Control) - PB & \\
\hline
\end{tabular}




\begin{tabular}{|c|c|c|}
\hline Mã hóa & $\begin{array}{l}\text { Biến quan sát của các nhân tố } \\
\text { Nhận thức dễ sử dụng (Perceived Ease of Use) - PE }\end{array}$ & -Nguồn \\
\hline PB1 & $\begin{array}{l}\text { Tôi có thể hoàn thành các hoạt động học tập của mình bằng dịch vụ } \\
\text { học trực tuyến nếu tôi chỉ có hướng dẫn sử dụng dịch vụ để tham } \\
\text { khảo }\end{array}$ & Roca et al. (2006) \\
\hline PB2 & $\begin{array}{l}\text { Tôi có thể hoàn thành các hoạt động học tập của mình bằng dịch vụ } \\
\text { học trực tuyến nếu tôi chưa bao giờ sử dụng một dịch vụ như vậy } \\
\text { trước đây }\end{array}$ & Roca et al. (2006) \\
\hline PB3 & $\begin{array}{l}\text { Tôi có thể sử dụng dịch vụ học trực tuyến mà không cần sự giúp đỡ } \\
\text { của bất kỳ ai }\end{array}$ & Wu et al. (2008) \\
\hline PB4 & $\begin{array}{l}\text { Tôi cảm thấy tự tin khi trao đổi thông tin với người khác trong các } \\
\text { diễn đàn thảo luận học trực tuyến }\end{array}$ & Roca et al. (2006) \\
\hline PB5 & $\begin{array}{l}\text { Tôi cảm thấy tự tin khi tải xuống các tài liệu từ dịch vụ học trực trực } \\
\text { tuyến }\end{array}$ & $\begin{array}{l}\text { Roca et al. (2006), Sun } \\
\text { et al. (2008) }\end{array}$ \\
\hline \multirow[t]{2}{*}{ PB6 } & Tôi cảm thấy tự tin khi đính kèm tập tin trong dịch vụ học trực tuyến & Roca et al. (2006) \\
\hline & Sự hài lòng (Satisfaction) - SAT & \\
\hline SAT1 & Tôi hài lòng với hiệu suất đạt được từ dịch vụ học trực tuyến & $\begin{array}{l}\text { Roca et al. (2006), Sun } \\
\text { et al. (2008) }\end{array}$ \\
\hline SAT2 & $\begin{array}{l}\text { Quyết định của tôi khi sử dụng dịch vụ học trực tuyến là một quyết } \\
\text { định khôn ngoan }\end{array}$ & $\begin{array}{l}\text { Roca et al. (2006), Sun } \\
\text { et al. (2008) }\end{array}$ \\
\hline SAT3 & Tôi hài lòng với trải nghiệm sử dụng dịch vu học trực tuyến & $\begin{array}{l}\text { Roca et al. (2006), Sun } \\
\text { et al. (2008) }\end{array}$ \\
\hline SAT4 & Tôi rất hài lòng với dịch vụ học tập trực tuyến & Sun et al. (2008) \\
\hline
\end{tabular}

Nguồn: tổng hợp tù̀ nghiên cúu của Roca et al.,2006; Pham et al., 2019; Sun et al., 2008; Wu et al., 2008

\subsection{Cõ̃ mẫu và cách thức khảo sát}

Tiến hành lấy mẫu với kích thước mẫu tối thiểu để sử dụng cho phân tích nhân tố khám phá EFA theo Hair et al. (1998) là $n=5^{*} 47=235$ để đảm bảo kích thước mẫu tối thiểu với 47 biến quan sát. Theo Green (1991), kích thước mẫu tối thiểu cho phân tích mô hình hồi quy với 8 biến độc lập được tính là $\mathrm{n}=50+8 * 8=114$. Ở sự kết hợp này, kích thước mẫu cần thiết là 300 để đề phòng những trường hợp phiếu trả lời không hợp lệ sẽ được loại bỏ sau khi sàn lọc. Phương pháp lấy mẫu thuận tiện và lấy mẫu theo kiểu phát triển mầm (quả bóng tuyết) được áp dụng để thu thập dữ liệu sơ cấp. Các đối tượng được khảo sát là những người đã hoặc đang tham gia khóa học trực tuyến. Nội dung câu hỏi gạn lọc được đưa vào bảng câu hỏi khảo sát nhằm xác định được đúng đối tượng cần thu thập thông tin. Đối với hình thức khảo sát trực tuyến, đường link khảo sát được gửi qua email, zalo nhờ vào sự hỗ trợ và giới thiệu của một số giảng viên - hiện là cố vấn học tập của sinh viên có tham gia hình thức học tập trực tuyến, sau đó nhờ giới thiệu người được khảo sát tiếp theo thông qua hình thức chia sẻ. Thêm vào đó, khảo sát trực tiếp cũng được thực hiện bằng cách in bảng câu hỏi ra và đi đến các trường học. Cụ thể, dữ liệu được thu thập từ các bạn sinh viên đang có các hoạt động tự học tại thư viện hoặc tại các khu vực sảnh chờ trong khuôn viên trường thông qua sự hỗ trợ của đồng nghiệp. Kết quả thu về nhận được nhiều phản hồi tích cực với 100 phiếu trả lời trực tuyến và 200 phiếu trực tiếp, dữ liệu sau khi thu thập được làm sạch thì có 267 phiếu trả lời hợp lệ và 33 phiếu trả lời không hợp lệ. Bên cạnh đó phương pháp thu thập thông tin thứ cấp được thực hiện thông qua các bài viết được xuất bản, các báo cáo nghiên cứu trước được công bố trên các trang web có uy tín từ các tổ chức trong và ngoài nước để có được những cơ sở lý thuyết tổng quan về hình thức học trực tuyến và sự hài lòng của người dùng.

\subsection{Phương pháp phân tích dữ liệu}

Sau khi sàn lọc và có được các dữ liệu chính thức từ các phương thức thu thập, nhóm tác giả tiến hành thống kê mô tả để tóm tắt dữ liệu và một số thông tin của mẫu như: giới tính, chuyên ngành đang theo học, trường theo học, năm học. Trong tổng 267 mẫu có 114 người là nam và 153 là nữ đang theo học tại một số trường đại học đã và đang triển khai hình thức học tập trực tuyến như: Đại học Kinh Tế Thành phố Hồ Chí Minh TP.HCM (15\%); Đại học Mở TP.HCM (13,5\%); Đại học Công Nghiệp TP.HCM (19,5\%); Đại học Tài Chính - Marketing (10,5\%); Đại học Công Nghệ TP.HCM (11,6\%); Đại học Văn Lang (9,4\%); Đại học Công Nghiệp Thực Phẩm TP.HCM (7,1\%); Đại học Lao Động - Xã Hội Cơ sở II $(7,5 \%)$ và Đại học Tôn Đức Thắng $(6 \%)$. Các chuyên ngành mà sinh viên đang theo học ghi nhận 
từ dữ liệu là quản trị kinh doanh, marketing, tài chính ngân hàng, kế toán, quản trị nguồn nhân lực, kinh doanh quốc tế, quản trị nhà hàng khách sạn và một số chuyên ngành khác. Ngoài ra, phần lớn các đối tượng được khảo sát là sinh viên năm hai và năm ba.

Sau đó, một số kĩ thuật phân tích được nhóm sử dụng bao gồm: kiểm định độ tin cậy thang đo bằng hệ số Cronbach's Alpha, phân tích nhân tố khám phá EFA, phân tích hồi quy tuyến tính để biết được các biến độc lập có sự tương quan tuyến tính với biến phụ thuộc hay không, sự tương quan đó tích cực hay tiêu cực và nó có ý nghĩa thống kê trong tổng thể hay không.

\section{KẾT QUẢ VÀ THẢO LUẬN}

\subsection{Kiểm định độ tin cậy Cronbach's Alpha}

Ngoài trừ các mục hỏi PU5, PU6 và IN6 có hệ số tương quan biến tổng nhỏ hơn 0,3 , kết quả phân tích cho thấy các mục hỏi trong cùng thang đo đều có các hệ số tương quan tổng lớn hơn 0,3 đạt được điều kiện cần thỏa mãn và hệ số Cronbach's Alpha tổng đều lớn hơn 0,7 (Bảng 2). Vì vậy, có thế kết luận rằng thang đo độc lập và phụ thuộc được sử dụng trong nghiên cứu là phù hợp và đáng tin cậy, các biến quan sát này đều được sử dụng trong phần phân tích nhân tố khám phá EFA tiếp theo.

\section{Bảng 2. Kết quả kiểm định độ tin cậy thang đo}

\begin{tabular}{lcc}
\hline Thang đo & Số mục hỏi & Hệ số Cronbach's Alpha \\
\hline Nhận thức sự hữu ích & 5 & 0,869 \\
Nhận thức dễ sử dụng & 4 & 0,890 \\
Chất lượng thông tin & 5 & 0,845 \\
Chất lượng hệ thống & 5 & 0,878 \\
Giảng viên hướng dẫn & 5 & 0,817 \\
Dịch vụ hỗ trợ & 5 & 0,884 \\
Chuẩn chủ quan & 4 & 0,894 \\
Kiểm soát nhận thức hành vi & 6 & 0,891 \\
Sự hài lòng & 4 & 0,908 \\
\hline
\end{tabular}

Nguồn: kết quả xủ lý số liệu khảo sát năm 2020

\subsection{Phân tích nhân tố khám phá EFA}

Thực hiện phân tích nhân tố khám phá cho thang đo các biến độc lập, kết quả ở lần phân tích cuối cùng thể hiện các tham số thống kê đều đạt yêu cầu (Bảng 3). Cụ thể, hệ số KMO =0,892 > 0,5 chứng tỏ phân tích có sự phù hợp và có ý nghĩa thống kê; giá trị sig. trong kiểm định Bartlett's $<0,05$ thể hiện các mục hỏi có tương quan với nhau; hệ số tải nhân tố đều lớn hơn 0,5 (ngoài trừ PE6 đã được loại bỏ). Chỉ số Eigenvalue (đại diện cho phần biến thiên được giải thích bởi mỗi nhân tố) bằng $1,291>1$ nên
8 nhân tố được rút trích có ý nghĩa tóm tắt thông tin tốt nhất. Đồng thời, tổng phương sai trích 68,393 cho biết 8 nhân tố này giải thích được $68,393 \%$ sự biến thiên của dữ liệu. Các nhóm nhân tố bao gồm: Nhận thức kiểm soát hành vi (PB5, PB4, PB2, PB1, PB6, PB3); Dịch vụ hỗ trợ (SS2, SS3, SS4, SS5, SS1); Chất lượng hệ thống (SY3, SY2, SY4, SY1, SY5); Chất lượng thông tin (IN3, IN2, IN4, IN1, IN5); Nhận thức dễ sử dụng (PE2, PE3, PE1, PE4, $\mathrm{PE} 5)$; Chuẩn chủ quan (SN2, SN3, SN1, SN4); Nhận thức sự hữu ích (PU2, PU3, PU1, PU4); Giảng viên hướng dẫn (ID4, ID5, ID3, ID2, ID1). 
Bảng 3. Kết quả phân tích nhân tố biến độc lập

\begin{tabular}{|c|c|c|c|c|c|c|c|c|}
\hline \multirow{2}{*}{ Chỉ tiêu } & \multicolumn{8}{|c|}{ Nhân tố } \\
\hline & X1 & $\mathbf{X} 2$ & $\mathbf{X 3}$ & X4 & X5 & X6 & $\mathbf{X} 7$ & $\mathrm{X8}$ \\
\hline PB5 & 0,789 & & & & & & & \\
\hline PB4 & 0,761 & & & & & & & \\
\hline PB2 & 0,715 & & & & & & & \\
\hline PB1 & 0,702 & & & & & & & \\
\hline PB6 & 0,697 & & & & & & & \\
\hline PB3 & 0,675 & & & & & & & \\
\hline $\mathrm{SS} 2$ & & 0,824 & & & & & & \\
\hline SS3 & & 0,760 & & & & & & \\
\hline $\mathrm{SS} 4$ & & 0,727 & & & & & & \\
\hline SS5 & & 0,653 & & & & & & \\
\hline $\mathrm{SS} 1$ & & 0,647 & & & & & & \\
\hline SY3 & & & 0,875 & & & & & \\
\hline SY2 & & & 0,840 & & & & & \\
\hline SY4 & & & 0,785 & & & & & \\
\hline SY1 & & & 0,775 & & & & & \\
\hline SY5 & & & 0,745 & & & & & \\
\hline IN3 & & & & 0,875 & & & & \\
\hline IN2 & & & & 0,872 & & & & \\
\hline IN4 & & & & 0,738 & & & & \\
\hline IN1 & & & & 0,720 & & & & \\
\hline IN5 & & & & 0,599 & & & & \\
\hline PE2 & & & & & 0,794 & & & \\
\hline PE3 & & & & & 0,785 & & & \\
\hline PE1 & & & & & 0,780 & & & \\
\hline PE4 & & & & & 0,670 & & & \\
\hline PE5 & & & & & 0,546 & & & \\
\hline SN2 & & & & & & 0,828 & & \\
\hline SN3 & & & & & & 0,813 & & \\
\hline SN1 & & & & & & 0,726 & & \\
\hline SN4 & & & & & & 0,718 & & \\
\hline PU2 & & & & & & & 0,822 & \\
\hline PU3 & & & & & & & 0,771 & \\
\hline PU1 & & & & & & & 0,752 & \\
\hline PU4 & & & & & & & 0,626 & \\
\hline ID4 & & & & & & & & 0,789 \\
\hline ID5 & & & & & & & & 0,740 \\
\hline ID3 & & & & & & & & 0,740 \\
\hline ID2 & & & & & & & & 0,718 \\
\hline ID1 & & & & & & & & 0,683 \\
\hline Hệ số KMO & & & & & & & & 0,892 \\
\hline Hệ số Sig (Bartlett's Test of Sphericity) & & & & & & & & 0,000 \\
\hline Giá trị Eigenvalues & 11,577 & 4,162 & 2.747 & 2.117 & 1.766 & 1.586 & 1.428 & 1.291 \\
\hline Mức độ giải thích của nhân tố (\%) & 10,693 & 9,004 & 8,823 & 8,395 & 8,366 & 7,865 & 7,628 & 7,619 \\
\hline Mức độ giải thích tích lũy (\%) & 10,693 & 19,6982 & 28,520 & 36,915 & 45,281 & 53,145 & 60,774 & 68,393 \\
\hline
\end{tabular}

Nguồn: kết quả xủ lý số liệu khảo sát năm 2020

Tương tự, các mục hỏi của biến phụ thuộc sau khi được kiểm định độ tin cậy thang đo bằng hệ số Cronbach's Alpha cũng được thực hiện phân tích nhân tố với kết quả cho thấy: Kiểm định Barlett's với giá trị Sig. $<0,05$ thể hiện các mục hỏi trong biến quan sát có sự tương quan với nhau. Hệ số
$\mathrm{KMO}=0,819>0,5$ chứng tỏ phân tích nhân tố thích hợp với dữ liệu nghiên cứu. Giá trị phương sai trích $=78,487$ và tất cả các mục hỏi đều có hệ số tải nhân tố lớn hơn 0,5 nên đạt yêu cầu. Như vậy thang đo "Sự hài lòng" đạt được giá trị hội tụ. 
Bảng 4. Kết quả phân tích nhân tố biến phụ thuộc

Tiêu chí Hệ số tải nhân tố

SAT2

SAT3

SAT1

Hệ số KMO

Hệ số Sig (Bartlett's Test of Sphericity)

Giá trị Eigenvalues

Mức độ giải thích (\%)

Nguồn: kết quả xủ lý số liệu khảo sát năm 2020

\subsection{Phân tích hồi quy}

Trong Bảng 5 , tất cả 8 nhân tố đều có giá trị mức ý nghĩa Sig. $<0,05$ nên có sự tương quan với sự hài lòng khi độ tin cậy là $95 \%$. $\mathrm{R}^{2}$ hiệu chỉnh đạt 0,667 , điều này nói lên có $66,7 \%$ sự thay đổi của sự hài lòng được giải thích bởi 8 nhân tố độc lập trong mô hình nghiên cứu, 33,3\% còn lại là do các tác động của các yếu tố khác. Kiểm định $\mathrm{F}$ sử dụng trong phân tích phương sai (ANOVA) của phân tích hồi quy đạt giá trị Sig. $<0,05$ điều này nói lên ý nghĩa mô hình lý thuyết phù hợp với dữ liệu thực tế.

\section{Bảng 5. Kết quả kiểm định mô hình của các nhân tố ảnh hưởng đến sự hài lòng}

\begin{tabular}{lccr}
\hline Nhân tố & Beta & Sig. & VIF \\
\hline Nhận thức kiểm soát hành vi & 0,199 & 0,000 & 1,963 \\
Dịch vụ hỗ trợ & 0,146 & 0,004 & 1,965 \\
Chất lượng hệ thống & 0,096 & 0,012 & 1,158 \\
Chất lượng thông tin & 0,101 & 0,010 & 1,206 \\
Nhận thức dễ sử dụng & 0,175 & 0,000 & 1,890 \\
Chuẩn chủ quan & 0,165 & 0,001 & 1,797 \\
Nhận thức sự hữu ích & 0,229 & 0,000 & 1,985 \\
Giảng viên hướng dẫn & 0,084 & 0,034 & 1,236 \\
\hline $\mathrm{R}^{2}$ & & & 0,677 \\
$\mathrm{R}^{2}$ hiệu chỉnh & & & 0,667 \\
Thống kê F (sig) & & & 0,000 \\
\hline
\end{tabular}

Nguồn: kết quả xủ lý số liệu khảo sát năm 2020

Dựa vào phương trình hồi quy cho thấy các hệ số hồi quy đều có giá trị lớn hơn 0 nên kết luận rằng tất cả các nhân tố độc lập được đưa vào phân tích đều có sự tác động thuận chiều đến biến phụ thuộc. Các nhân tố độc lập bao gồm nhận thức dễ sử dụng, nhận thức sư hưu ich, chất lượng thông tin, chất luợng hệ thống, giảng viên huớng dẫn, dịch vu hỗ trợ, chuẩn chủ quan và nhận thức kiểm soát hành vi đều có sự tác động tích cực và đáng kể đến biến phụ thuộc là sụ hài lòng.

Dựa vào giá trị của hệ số beta (Bảng 5), nhân tố nhận thức sự hữu ích có sự ảnh hưởng lớn nhất trong 8 nhân tố. Điều này phù hợp với nghiên cứu trước đây cho thấy rằng nhận thức sự hữu ích có vai trò quan trọng hơn nhận thức dễ sử dụng khi xem xét trong bối cảnh học tập trực tuyến. Cụ thể, người dùng quan tâm đển cách mà hệ thống học tập trực tuyến cung cấp thông tin và lợi ích hay hiệu quả mà nó mang đến (Roca et al., 2006; Sun et al., 2008).

Nhân tố có mức độ ảnh hưởng lớn thứ hai sau nhận thức sự hữu ích là nhận thức kiểm soát hành $v i$. Kết quả này cũng ủng hộ với các nghiên cứu Roca et al. (2006), Sun et al. (2008), Wu et al. (2008) khi có sự tương quan thuận đối với sự hài lòng. Trên thực tế khi một sinh viên sử dụng dịch vụ học trực tuyến sẽ có những sự lo lắng về việc tương tác, thao tác với máy tính và trang web học trực tuyến. Sự lo lắng càng cao thì sinh viên càng không thể xử lý tốt các thông tin hay yêu cầu khi sử dụng, nên việc sinh viên nhận thức được khả năng hành vi của mình hay sinh viên tự tin trao đổi thông tin, thao tác trên hệ thống sẽ tạo cho sinh viên thái độ tích cực hơn đối với hệ thống học trực tuyến. 
Kế đến, nhận thức dễ sư dụng có sự tác động đáng kể và mạnh thứ ba đến sự hài lòng. Từ góc nhìn thực tế liên quan đến học tập trực tuyến, việc sinh viên dễ dàng tiếp cận, dễ sử dụng hệ thống, dễ tìm kiếm thông tin sẽ kích thích và mang lại những cảm xúc tích cực từ đó khuyến khích người học hào hứng tham gia khóa học hơn. Kết quả này cũng đã kiểm chứng trong nghiên cứu của Roca et al. (2006). Đặc biệt, trong nghiên cứu của Tarhini et al. (2013) thể hiện nhận thức dễ sử dụng có sự ảnh hưởng mạnh nhất đến sự hài lòng. Việc dễ dàng sử dụng sẽ giúp người dùng tập trung vào các thông tin hơn tạo nên hiệu quả cao. Tuy nhiên, kết quả về mối tương quan giữa nhân tố chuẩn chủ quan và sự hài lòng lại không nhất quán so với nghiên cứu của Roca et al. (2006). Theo đó, sự tác động từ gia đình, bạn bè, người thân và người hướng dẫn thực sự có ý nghĩa đối việc củng cố niềm tin đặc biệt là nhìn nhận được lợi ích từ công nghệ dẫn đến việc sử dụng hình thức học tập trực tuyến khi trong bối cảnh thực hiện phòng, chống dịch phức tạp đã và đang diễn ra.

Thêm vào đó, Nhân tố dịch vu hỗ trợ cũng được đánh giá có sự ảnh hưởng tương quan thuận đến sự hài lòng của người học. Trong quá trình sử dụng hệ thống học trực tuyến, nhất định người học sẽ gặp những vấn đề mà tự bản thân họ không thể giải quyết được điều này cần có sự hỗ trợ kịp thời từ phía nhà trường hay người quản lý. Đáp ứng được các nhu cầu về lựa chọn khóa học, sự bảo mật, cách khắc phục sự cố hay các thủ tục hành chính nhanh chóng sẽ làm tăng sự hài lòng của sinh viên về hệ thống học trực tuyến hơn. Kết quả này một lần nữa ủng hộ quan điểm của Pham et al. (2019). Một khi sinh viên được hỗ trợ nhanh chóng, kịp thời trong quá trình sử dụng sẽ tạo sự tin tưởng, sự hài lòng khi những lợi ích của sinh viên có đều được nhà trường quan tâm.

Ngoài ra, chất lượng thông tin và chất lượng hệ thống là hai nhân tố quan trọng và đáng quan tâm. Bản thân người học không chỉ chú ý vào việc hệ thống học tập cũng cấp những thông tin gì, những thông tin đó có rõ ràng, dễ hiểu và phù hợp hay không mà còn đặc biệt quan tâm đến khả năng phản hồi nhanh chóng trong thời gian cao điểm, việc sắp xếp thông tin hiển thị và vấn đề bảo mật thông tin. Việc học trực tuyến dựa trên các thiết bị, phương tiện điện tử có sự kết nối mạng thì không thể nào thiếu đi tính năng hệ thống, hệ thống dịch vụ là nơi để người học học tập, hoàn thành các bài tập được giao nên chất lượng hệ thống là đều đáng quan tâm đến. Công nghệ thông tin phát triển mạnh mẽ, đòi hòi từ người dùng ngày càng cao nên chất lượng hệ thống càng được quan tâm để cải thiện phù hợp với nhu cầu người dùng. Điều này hoàn toàn phù hợp và cũng đã được ủng hộ trong một số nghiên cứu của của Pham et al. (2019), Mohammadi (2015) và Roca et al. (2006).

Và sau cùng, nhân tố giảng viên hướng dẫn được đánh giá là có sự ảnh hưởng đối với sự hài lòng của người học. Đối với hình thức học tập truyền thống phần lớn sự tương tác giữa người dạy với người học chủ yếu là giao tiếp trực tiếp, trong khi đối với hình thức học tập trực tuyến thì sự giao tiếp này là gián tiếp thông qua môi trường không gian "ảo" bằng cách sử dụng các công cụ, trang thiết bị điện tử có kết nối mạng internet. Sự tương tác này có phần hạn chế nếu như đường truyền không ổn định hoặc gặp những vấn đề không mong muốn khi kết nối. Vì vậy, bên cạnh kiến thức chuyên môn tốt thì giảng viên hướng dẫn đóng vai trò quan trọng trong việc khuyến khích sự tham gia tương tác và sự tích cực học tập của người học thông qua phương pháp giảng dạy, cách thức tổ chức bài học, bài tập, bài kiểm tra (Pham et al., 2019; Sun et al., 2008).

\section{KẾT LUẬN VÀ HÀM Ý QUẢN TRI}

\subsection{Kết luận}

Kết quả nghiên cứu đã làm rõ được những vấn đề liên quan đến hình thức học trực tuyến và sự hài lòng của người học thông qua mô hình nghiên cứu được đề xuất và kiểm định về mối tương quan giữa các nhân tố với sự hài lòng của người học. Theo đó, có mối quan hệ tương quan dương giữa nhận thức sự hữu ích; nhận thức dễ sử dụng, chất lượng thông tin; chất lượng hệ thống; giảng viên hướng dẫn; dịch vụ hỗ trợ; chuẩn chủ quan; kiểm soát nhận thức hành vi với sự hài lòng. Kêt quả này cũng cho thấy có sự phù hợp với thực tế và một lần nữa ủng hộ kết quả các nghiên cứu trước đây của Chiu et al. (2005), Roca et al. (2006), Sun et al. (2008), Tarhini et al. (2013), Vũ Thúy Hằng và Nguyễn Mạnh Tuân (2013), Mohammadi (2015), Pham et al. (2019). Sau khi kết hợp giữa lý thuyết và kết quả phân tích dữ liệu, nhóm tác đã có những đề xuất hàm ý quản trị nhằm giúp cho các cơ sở giáo dục đào tạo đã và đang triển khai hình thức học tập trực tuyến có thể nâng cao chất lượng dịch vụ, sự hài lòng của người học.

\subsection{Hàm ý quản trị}

Dựa trên kết quả phân tích, nội dung thảo luận trước đó, nghiên cứu đưa ra một số đề xuất hàm ý quản trị nhằm giúp hoàn thiện và nâng cao sự hài lòng của sinh viên hơn đối với hình thức học trực tuyến (e-leaning):

Các cơ sở đào tạo cần có những tài liệu và video hướng dẫn cách thức truy cập, thao tác và cách thức sử dụng các chức năng trong hệ thống dịch vụ trước 
khi người dùng bắt đầu khóa học. Ngoài ra, nhà trường cần quan tâm đến việc làm giảm đi tính phức tạp của hệ thống khi người dùng tương tác như: cần sắp xếp thông tin hiển thị hợp lý và dễ hiểu, giảm bớt sự hiển thị những hình ảnh không cần thiết trong hệ thống, đơn giản hóa thao tác nộp bài tập, tra cứu, tìm kiếm thông tin. Các thông tin được hiển thị trên hệ thống học trực tuyến hay các trang web cần phải chính xác, dễ dàng truy cập và tiếp cận.

Các trường đại học cần đáp ứng kịp thời các yêu cầu thông tin, lựa chọn các khóa học, các vấn đề về lỗi hệ thống, đăng nhập, truy cập lớp học và các thủ tục hành chính khác trước, trong và sau quá trình học tập qua hình thức học trực tuyến một cách nhanh chóng, chính xác và linh hoạt. Ngoài ra, bên cạnh tài liệu hướng dẫn chung và lưu ý khắc phục sự cố thường gặp thì các đơn vị đào tạo cũng cần phải duy trì việc hỗ trợ xuyên suốt để tránh tình trạng sinh viên gặp vấn đề khó khăn không giải quyết được.

Đảm bảo thông tin nhanh chóng và chuẩn xác thông qua tính năng thông báo của hệ thống được gửi qua email và SMS của người học. Xây dựng và cập nhật thường xuyên hệ thống học liệu điện tử để đảm bảo được tính phù hợp với bối cảnh cũng như sự đổi mới. Đồng thời, điều này giúp người học có thể tiếp cận mọi lúc mọi nơi tăng tính chủ động trong quá trình giảng dạy và học tập.

Trong những buổi giảng trực tuyến, nên ghi âm lại bài giảng để sau buổi học người học có thể nghe lại bằng cách truy cập vào hệ thống đã cấp quyền và phân quyền trong trường hợp người học chưa hoặc không nắm hết thông tin do vấn đề tốc độ đường truyền, tín hiệu âm thanh và một số các phát sinh ngoài ý muốn từ ngoại cảnh.

\section{3. Đề xuất hướng nghiên cứu tiếp theo}

Bên cạnh những kết quả đạt được, nghiên cứu cũng còn một số hạn chế nhất định. Quy mô mẫu tuy đạt yêu cầu về mặt thống kê tuy nhiên đối tượng khảo sát chỉ mới tập trung vào sinh viên ngành kinh tế tại khu vực TP.HCM. Vì vậy, hướng nghiên cứu kế tiếp nên có sự mở rộng về các khối ngành khác hoặc có sự mở rộng về quy mô không gian địa lý. Ngoài ra, kết quả phân tích ở những nghiên cứu sau nên có sự đối sánh sự hài lòng của đối tượng người học xét theo những đặc tính khác biệt về ngành học, không gian địa lý hoặc giữa các trường với nhau.

\section{TÀI LIỆU THAM KHẢO}

Ajzen, I. (1991). The theory of planned behavior. Organizational behavior and human decision processes, 50(2), 179-211.
Bùi Kiên Trung. (2016). Mối quan hệ giữa chất luọng dịch vu đào tạo vói sụ hài lòng và múc độ trung thành của sinh viên trong đào tạo tì xa $E$ Learning (luận án tiến sĩ kinh tế). Trường Đại học Kinh Tế Quốc Dân.

Chiu, C. M., Hsu, M. H., Sun, S. Y., Lin, T. C., \& Sun, P. C. (2005). Usability, quality, value and elearning continuance decisions. Computers \& education, 45(4), 399-416.

https://doi.org/10.1016/j.compedu.2004.06.001

Davis, F.D. (1989). Perceived usefulness, perceived ease of use, and user acceptance of information technology. MIS Quarterly, 13 (3), 319-340. https://doi.org/10.2307/249008

DeLone, W. H., \& McLean, E. R. (1992). Information systems success: The quest for the dependent variable. Information systems research, 3(1), 60-95. https://doi.org/10.1287/isre.3.1.60

DeLone, W. H., \& McLean, E. R. (2003). The DeLone and McLean model of information systems success: a ten-year update. Journal of management information systems, 19(4), 9-30. https://doi.org/10.1080/07421222.2003.11045748

Green, S. B. (1991). How many subjects does it take to do a regression analysis. Multivariate behavioral research, 26(3), 499-510. https://doi.org/10.1207/s15327906mbr2603_7

Hair, J. F., Black, W. C., Babin, B. J., Anderson, R. E., \& Tatham, R. L. (1998). Multivariate data analysis. Prentice Hall.

Harasim, L. (2006). A History of E-learning: Shift Happened. In Weiss, J., Nolan, J., Hunsinger, J., Trifonas, P. (Eds.), The International Handbook Of Virtual Learning Environments (pp. 59-94). Spinger, Dordrecht.

Huỳnh Đệ Thủ. (2019). Hệ thống đào tạo trực tuyến ELearning tại Trường Đại Học Kinh tế - Tài chính TP. Hồ Chí Minh: Nghiên cứu đánh giá và kiến nghị. Phát triển và hội nhập, 46(56), 100-105.

Innovation Center Denmark Seoul. (2014). Elearning in Korea: Overview of E-learning sector in Korea. http://kursobr.ru/images/elearning.pdf

Isik, O. (2008, November 22-25). E-learning satisfaction factors. In Proceedings of the 39th annual meeting of the decision sciences institute, Baltimore, Maryland, United States.

Kentnor, H. E. (2015). Distance education and the evolution of online learning in the United States. Curriculum and teaching dialogue, 17(1), 21-34.

Martínez-Argüelles, M. J., \& Batalla-Busquets, J. M. (2016). Perceived service quality and student loyalty in an online university. International 
Review of Research in Open and Distributed Learning, 17(4), 264-279.

Martín-Rodríguez, Ó., Fernández-Molina, J. C., Montero-Alonso, M. Á., \& González-Gómez, F. (2015). The main components of satisfaction with e-learning. Technology, Pedagogy and Education, 24(2), 267-277. https://doi.org/10.1080/1475939X.2014.888370

Mohammadi, H. (2015). Investigating users' perspectives on e-learning: An integration of TAM and IS success model. Computers in human behavior, 45, 359-374. https://doi.org/10.1016/j.chb.2014.07.044

Pham, L., Limbu, Y. B., Bui, T. K., Nguyen, H. T., \& Pham, H. T. (2019). Does e-learning service quality influence e-learning student satisfaction and loyalty? Evidence from

Vietnam. International Journal of Educational Technology in Higher Education. 16(1), 1-26. https://doi.org/10.1186/s41239-019-0136-3

Roca, J. C., Chiu, C. M., \& Martínez, F. J. (2006). Understanding e-learning continuance intention: An extension of the Technology Acceptance Model. International Journal of humancomputer studies, 64(8), 683-696. https://doi.org/10.1016/j.ijhcs.2006.01.003

Seddon, P. B. (1997). A respecification and extension of the DeLone and McLean model of IS success. Information systems research, 8(3), 240-253. https://doi.org/10.1287/isre.8.3.240

Sun, P. C., Tsai, R. J., Finger, G., Chen, Y. Y., \& Yeh, D. (2008). What drives a successful eLearning? An empirical investigation of the critical factors influencing learner satisfaction. Computers \& education, 50(4),
1183-1202.

https://doi.org/10.1016/j.compedu.2006.11.007

Tarhini, A., Hone, K. S., \& Liu, X. (2013). Factors affecting students' acceptance of e-learning environments in developing countries: a structural equation modeling approach. International Journal of Information and Education Technology, 3(1), 54-59.

Tổng cục Thống kê. (2018). Số sinh viên các trường đại hoc phân theo địa phuong. Retrieved November 20, 2020, from https:/www.gso.gov.vn/giao-duc/

Bộ Giáo dục và Đào tạo. (2020). Hội nghị trực tuyến về phòng, chống covid 19 và hướng dẫn dạy học trực tuyến. Retrieved November 20, 2020, from https://moet.gov.vn/tintuc/Pages/tin-hoat-dongcua-bo.aspx?ItemID $=6576$

Vũ Hữu Đức, 2020. Nghiên cứu về phương thức học tập, đào tạo dựa trên công nghệ thông tin (Elearning) trong giáo dục đại học và đào tạo trực tuyến mở dành cho đại chúng MOOCs (Massive Online Open Courses): Kinh nghiệm thế giới và ứng dụng tại Việt Nam. Đề tài NCKH cấp Quốc gia, mã KHGD/16-20.ĐT.043.

Vũ Thúy Hằng \& Nguyễn Mạnh Tuân. (2013). Tích hợp các yếu tố ảnh hưởng đến hài lòng của người học vào hệ thống e-Learning: một tình huống tại Trường Đại học Kinh tế-Luật. Tạp chí Khoa học Đại học Su Phạm Thành Phố Hồ Chí Minh, 53, 24-46.

Wu, J. H., Tennyson, R. D., \& Hsia, T. L. (2010). A study of student satisfaction in a blended elearning system environment. Computers \& Education, 55(1), 155-164. https://doi.org/10.1016/j.compedu.2009.12.012 